This item was submitted to Loughborough's Research Repository by the author.

Items in Figshare are protected by copyright, with all rights reserved, unless otherwise indicated.

\title{
Activation of a bis-(phenoxyimine) titanium (IV) catalyst using different aluminoxane co-catalysts
}

PLEASE CITE THE PUBLISHED VERSION

http://dx.doi.org/10.1002/masy.201500047

\section{PUBLISHER}

(c) Wiley-VCH Verlag

\section{VERSION}

AM (Accepted Manuscript)

\section{PUBLISHER STATEMENT}

This work is made available according to the conditions of the Creative Commons Attribution-NonCommercialNoDerivatives 4.0 International (CC BY-NC-ND 4.0) licence. Full details of this licence are available at: https://creativecommons.org/licenses/by-nc-nd/4.0/

\section{LICENCE}

CC BY-NC-ND 4.0

\section{REPOSITORY RECORD}

Romano, Dario, Sara Ronca, and Sanjay Rastogi. 2015. "Activation of a Bis-(phenoxyimine) Titanium (IV) Catalyst Using Different Aluminoxane Co-catalysts". figshare. https://hdl.handle.net/2134/18153. 


\title{
Activation of a Bis-(Phenoxyimine) Titanium (IV) Catalyst Using Different Aluminoxane Co-catalysts
}

\author{
Dario Romano*, ${ }^{1}$ Sara Ronca, ${ }^{1}$ Sanjay Rastogi ${ }^{1,2}$ \\ ${ }^{1}$ Department of Materials, Loughborough University, Ashby Road, LE11 \\ 3TU, Loughborough, United Kingdom \\ ${ }^{2}$ Research Institute, Teijin Aramid, Velperweg 76, Arnhem 6802ED, The \\ Netherlands \\ E-mail: D.Romano@lboro.ac.uk
}

Summary: The activation of a bis-phenoxyimine catalyst based on titanium (IV) using different aluminoxanes (MAO, PMAO and MMAO12) has been studied. The effect of a co-catalyst modifier (BHT) used in combination with the MAO has been also tested. In particular, the effect of the activation time between the catalyst and the different aluminoxanes has been taken into consideration. On increasing the activation time between catalyst and the different aluminoxanes and TMA-free MAO, differences in the catalyst activities have been observed. UHMWPEs having a reduced number of entanglements have been synthesized activating the FI catalyst with MAO and TMA-free MAO. The obtained reactor powders can be solid-state processed below the melting temperature in order to obtain high modulus/high tenacity tapes used for body armor and vehicle protection applications.

Keywords: UHMWPE, high performance polymers, rheology, activation, entanglements

\section{Introduction}

Since their discovery in the late 1990s by Fujita and co-workers, bis-phenoxyimine class of catalysts has attracted much attention due to their ease in synthesis and catalyst modification. ${ }^{[1,2]}$ Several articles ${ }^{[3,4]}$ and reviews ${ }^{[5,6]}$ have been published where the catalytic behavior and mechanistic studies have been performed using this class of catalysts. Particular attention has been posed for the fluorinated aryl derived due to their capabilities to promote the synthesis of Ultra-High Molecular Weight Polyethylene (UHMWPE) due to a reduced $\beta$-hydrogen chain termination process. In addition, these catalysts are capable to synthesize UHMWPE with a "quasi living" behavior, making them suitable catalyst for fundamental studies. ${ }^{[7]}$ Using the bis[N-(3-tertbutylsalicylidene)pentafluoroanilinato] titanium (IV) dichloride (FI) catalyst, Rastogi et al. have synthesize UHMWPE having a reduced number of entanglements between the chains (disentangled-UHMWPE). ${ }^{[8]}$ The base concept of the synthesis of disentangle-UHMWPE 
is a fast crystallization rate over the polymerization rate, allowing the growing chain to crystallize faster, thus reducing the probability of self-entangle or entanglement formation with neighbor chains. The use of homogeneous catalyst with respect to heterogeneous ones greatly reduces the probability to entangle due to a good separation in the reaction media of the active sites (and consequently the growing chains). ${ }^{[9]}$ Industrially, commercially available entangled UHMWPE can be processed into fibers by dissolving small amount of powder into large volume of aromatic solvent (typically 5/95 volume ratio respectively). In this process of solution spinning, the entanglement density is reduced below a critical limit. The resultant viscous solution is stretched into fibers and the solvent is removed and recycled. ${ }^{[10,11]}$ The main advantage of the adopted approach in this publication is the control of the entanglement density during synthesis thus avoiding the cumbersome use of solvent and its recovery. The adopted controlled synthesis route makes the processing of the reactor powder more environmental friendly. The reactor powder obtained can be solid-state processed below the melting temperature in order to obtain high modulus/high tenacity tapes without the use of any solvent with clear economic and environmental advantages. Such tapes can be used in making light weight composites meeting the requirements of body and vehicle protection. ${ }^{[8]}$ Following studies demonstrated the dependence of the mechanical properties to the molecular weight and entanglement density of the polymers produced and the main chain termination processes. $^{[12]}$ Hence, the understanding of the processes involved during the polymerization is of important relevance in both academic and industrial applications.

In this study, the catalyst activation and possible catalyst deactivation processes have been investigated using the FI catalyst in the polymerization of ethylene into disentangleUHMWPE. The use of a co-catalyst modifier in combination with methylaluminoxane (MAO) is a well-known chemical treatment to scavenge the trimethylaluminum (TMA) in equilibrium with the MAO. ${ }^{[13,14]}$ The effect of a co-catalyst modifier (2,6-di-tert-butyl-4methylphenol, BHT) in the activation procedure is also established. The influence of the catalyst activation time in the presence of different aluminoxane co-catalysts is also addressed. This study aims to increase the knowledge on the deactivation and chain termination processes of the FI catalyst upon activation by MAO and/or different aluminoxane-based co-catalysts. 


\section{Materials}

All manipulations of air and moisture-sensitive compounds are performed under nitrogen or argon atmosphere using standard high-vacuum Schlenk techniques or in an MBraun 350 glovebox. Ethylene (grade 3.5) is purchased from BOC, bis[N-(3-tertbutylsalicylidene)pentafluoroanilinato]titanium (IV) dichloride is received from MCat and used as received. Toluene (anhydrous 99.9\%) and 2,6-di-tert-butyl-4-methylphenol $(\geq 99.0 \%)$ are obtained from Sigma-Aldrich ${ }^{\circledR}$ and used as received. Methylaluminoxane (10 wt. \% toluene solution) is supplied from Albemarle ${ }^{\circledR}$ and used as received. PMAO and MMAO12 are purchased from AkzoNobel and used as received. BASF Irganox 1010 is added to the polymer as antioxidant.

\section{Polymerization}

An overnight pre-dried 1.5 l cylindrical jacketed glass reactor vessel having a five-necked flanged lid, equipped with a thermometer probe, stainless steel feeding tube, gas release system, injection septum and a stopper cone is kept overnight at $130{ }^{\circ} \mathrm{C}$. The reactor vessel is backfilled with nitrogen and, after at least 3 cycles of vacuum/nitrogen, is charged with $0.75 \mathrm{l}$ of anhydrous toluene at room temperature and dry nitrogen is bubbled through the solvent for $\sim 30 \mathrm{~min}$ under stirring. The solvent temperature is set at $10^{\circ} \mathrm{C}$ and controlled by a feedback loop control thermostat Thermo-Scientific NESLAB RTE7. After the thermal equilibration is reached, a desired amount of aluminoxane solution is added (if BHT is used, the aluminoxane solution is previously reacted with $0.8 \mathrm{~g}$ of BHT for 30 minutes). Using a Büchi BPC6002 press flow gas controller unit, the nitrogen stream is replaced with ethylene gas at 1.1 bar absolute pressure. After the solvent saturation and thermal equilibration is obtained, the polymerization is initiated by addition of the FI catalyst $(5 \mathrm{mg})$ dissolved in toluene $(1 \mathrm{ml})$ and the desired amount of aluminoxane solution (if BHT is used, the aluminoxane solution is reacted for 30 minutes with $0.2 \mathrm{~g}$ of BHT). After 1 hour, the reaction is terminated by addition of methanol. The synthesized polymer is washed and filtered. In order to prevent degradation of the polymer during the long rheological experiments $(>24 \mathrm{~h})$, the nascent powder is mixed with antioxidant (0.7 wt. \%, Irganox 1010). The polymer obtained is dried in a vacuum oven at $40^{\circ} \mathrm{C}$ overnight.

\section{Rheological characterization}


The nascent powder sample is hot-compressed into a disc of $\sim 35 \mathrm{~mm}$ diameter and thickness $0.6-0.7 \mathrm{~mm}$ at a fixed temperature of $125^{\circ} \mathrm{C}$, under a maximum force of 20 tons for an average time of $20 \mathrm{~min}$. Using a punching device, $12 \mathrm{~mm}$ diameter discs are cut for rheological studies. Rheological experiments are performed using a TA Instrument Ares G2 or LS2. To prevent oxidation during the long experiments, the temperature is controlled by a convection oven under a nitrogen environment. The sample is placed between parallel plates at an initial temperature of $110{ }^{\circ} \mathrm{C}$. After thermal stabilization, the temperature is increased to $130{ }^{\circ} \mathrm{C}$ at $30{ }^{\circ} \mathrm{C} / \mathrm{min}$ under a compression force of $1 \mathrm{~N}$. After thermal stabilization, the sample is brought to the melt state at $160{ }^{\circ} \mathrm{C}$ at $10{ }^{\circ} \mathrm{C} / \mathrm{min}$ while maintaining a constant compression force of $4 \mathrm{~N}$. After 90 seconds of thermal equilibration, an oscillatory amplitude sweep test is carried out to determine the range of oscillatory strains in the linear viscoelastic region at a fixed frequency of $10 \mathrm{rad} / \mathrm{s} .{ }^{[15]} \mathrm{To}$ follow changes in the elastic modulus of the polymer melt an oscillatory time sweep test is performed. ${ }^{[9]}$ The test is carried out at a fixed frequency of $10 \mathrm{rad} / \mathrm{s}$ and a strain of $\sim 0.3 \%$, well within the linear viscoelastic regime. To determine the molecular weight of the polymer, after reaching the storage modulus plateau, small amplitude oscillatory frequency sweep test at a fixed strain of $0.3 \%$ is performed. Using the orchestrator software available on the rheometer, the molecular weight and molecular weight distribution is determined by means of the method developed by Mead. ${ }^{[16]}$

\section{Results and Discussion}

The following results highlight the catalyst activity and the activation capability of the MAO (and trimethylaluminum-free MAO) in the polymerization of ethylene promoted by the bis[ $N$-(3-tert-butylsalicylidene)pentafluoroanilinato] titanium (IV) dichloride catalyst. The activation capabilities of the MAO (and trimethylaluminum-free MAO) have been evaluated by increasing the activation contact time between catalyst and co-catalyst prior to injection into the reactor containing ethylene. Table 1 summarizes the ethylene polymerization data using the FI catalyst activated with MAO and in combination with BHT.

Table 1 Ethylene polymerization data using MAO and in combination with a co-catalyst modifier in different activation procedures.

\begin{tabular}{|l|l|l|l|l|l|l|}
\hline Run & $\begin{array}{l}\text { Co-catalyst as } \\
\text { Scavenger }\end{array}$ & $\begin{array}{l}\text { Co-catalyst as } \\
\text { Activator }\end{array}$ & $\begin{array}{l}\text { Activation Time } \\
\text { (min) }\end{array}$ & $R p^{a}$ & $\begin{array}{l}M_{\mathrm{w}} \\
\left(* 10^{6}\right.\end{array}$ & MWD \\
\hline
\end{tabular}




\begin{tabular}{|l|l|l|l|l|l|l|}
\hline & & & & & g/mol $)$ & \\
\hline 1 & $4 \mathrm{ml}$ of MAO & $1 \mathrm{ml}$ of MAO & 0 & 4000 & 9.3 & 3.3 \\
\hline 2 & $4 \mathrm{ml}$ of MAO & $1 \mathrm{ml}$ of MAO & 15 & 2650 & 11.2 & 7.8 \\
\hline 3 & $4 \mathrm{ml}$ of MAO & $1 \mathrm{ml}$ of MAO & 30 & 1450 & n.d. & n.d. \\
\hline 4 & $4 \mathrm{ml}$ of MAO & $\begin{array}{l}1 \mathrm{ml} \text { of MAO } \\
+0.2 \mathrm{~g} \text { of BHT }\end{array}$ & 0 & 4150 & 7.5 & 3.7 \\
\hline 5 & $4 \mathrm{ml}$ of MAO & $\begin{array}{l}1 \mathrm{ml} \text { of MAO } \\
+0.2 \mathrm{~g} \text { of BHT }\end{array}$ & 15 & 4200 & 7.1 & 3.5 \\
\hline 7 & $\begin{array}{l}4 \mathrm{ml} \text { of MAO } \\
0.8 \mathrm{~g} \text { of BHT }\end{array}$ & $\begin{array}{l}1 \mathrm{ml} \text { of MAO } \\
+0.2 \mathrm{~g} \text { of BHT }\end{array}$ & 30 & 3700 & 6.6 & 3.5 \\
\hline
\end{tabular}

Other conditions: Al/Ti molar ratio, 1200; catalyst, $6.2 \pm 0.2 \mu \mathrm{mol}$; temperature, $10{ }^{\circ} \mathrm{C}$; reaction volume, $750 \mathrm{ml}$ of toluene; reaction time, $60 \mathrm{~min}$; ethylene pressure, $1.1 \mathrm{~atm} .{ }^{\text {a }}$ $\mathrm{kg}_{\mathrm{PE}} / \mathrm{mol}_{\mathrm{Ti}} * \mathrm{~atm} *$ hour.

The relative low yield of the Run 3 leads to high catalytic ashes content and the rheological response of the polymer was affected. For this reason the characterization of this polymer is excluded in the discussion. From the polymerizations data in table 1 it is clear that the catalyst activity depends on the activation time when the activator is MAO only. In the case of the MAO (having TMA), the use of BHT also influences the catalyst activation capability of the activator. For the activation using MAO, when increasing the catalyst activation time prior to injection into the reactor, the catalyst activity decreases of $64 \%$ after 30 minutes of activation time (Runs 1-3). Using the BHT solely in the activation step (Runs 4-6) the catalyst activity decreases of 10\% (within the experimental error) after 30 minutes of activation time. Figure 1 summarizes the data regarding the activation with MAO and MAO+BHT. The data confirm that in the presence of BHT, the polymerization rate is independent of the activation time. 


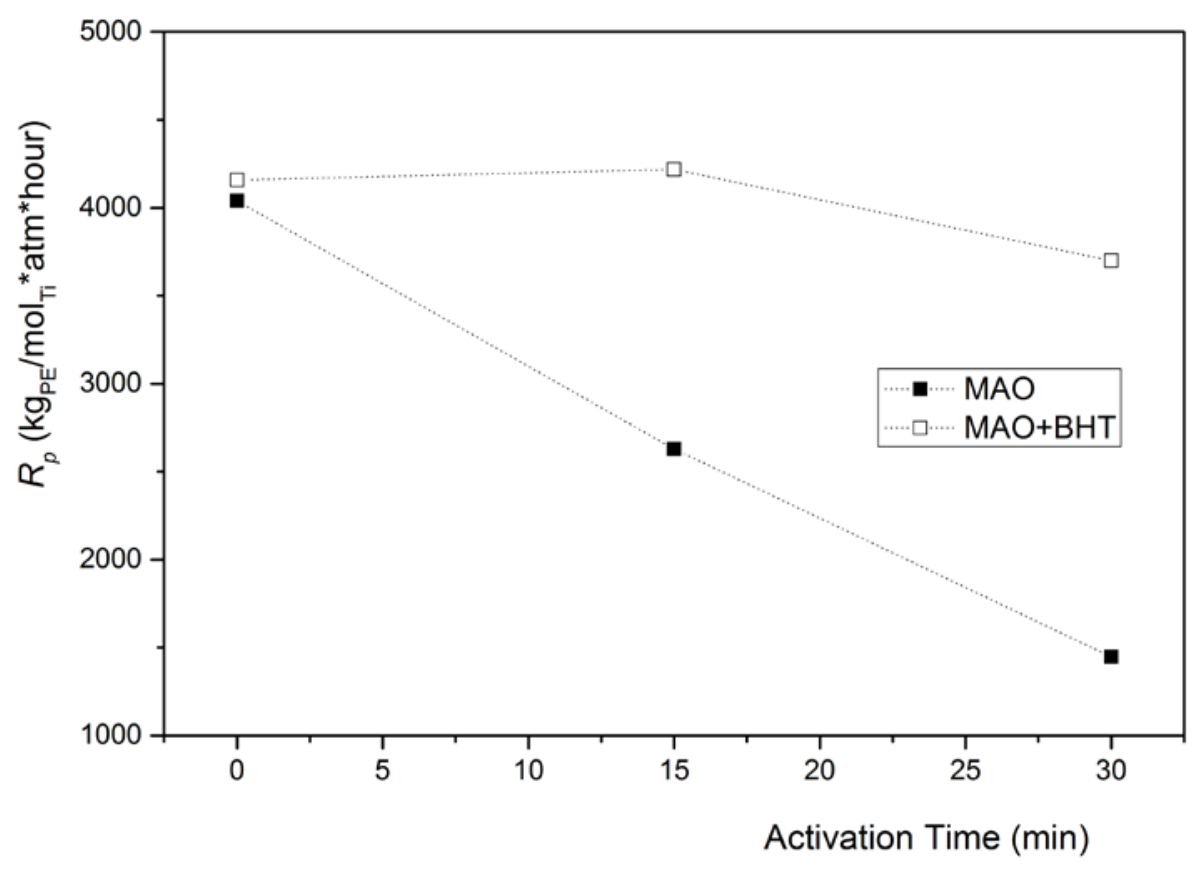

Figure 1: Catalyst activity as a function of activation time for the activation using MAO (filled squares) and MAO+BHT (unfilled squares). The dotted lines between points are drawn for eye guidance only.

It is plausible to think that increasing the activation time (contact time between the catalyst and MAO), the TMA can interact with the catalyst leading to deactivation. Prior findings suggest a possible formation of dimethyl bridged species between the TMA and the catalyst, also called dormant sites, or reduction of Ti (IV) into Ti (III) involving the MAO or catalyst ligand transfer to the TMA. ${ }^{[17,18,19]}$ It is reasonable to think that when the concentration of TMA is reduced by the use of BHT in the activation, the above catalyst deactivations are suppressed in the considered time range. An insight on the role of TMA on the molecular weight capability of this catalytic system has been presented previously by our research group. ${ }^{[20]}$

Figure 2 shows the ethylene uptake profile normalized for the amount of catalyst as a function of polymerization time for different activation procedures. 


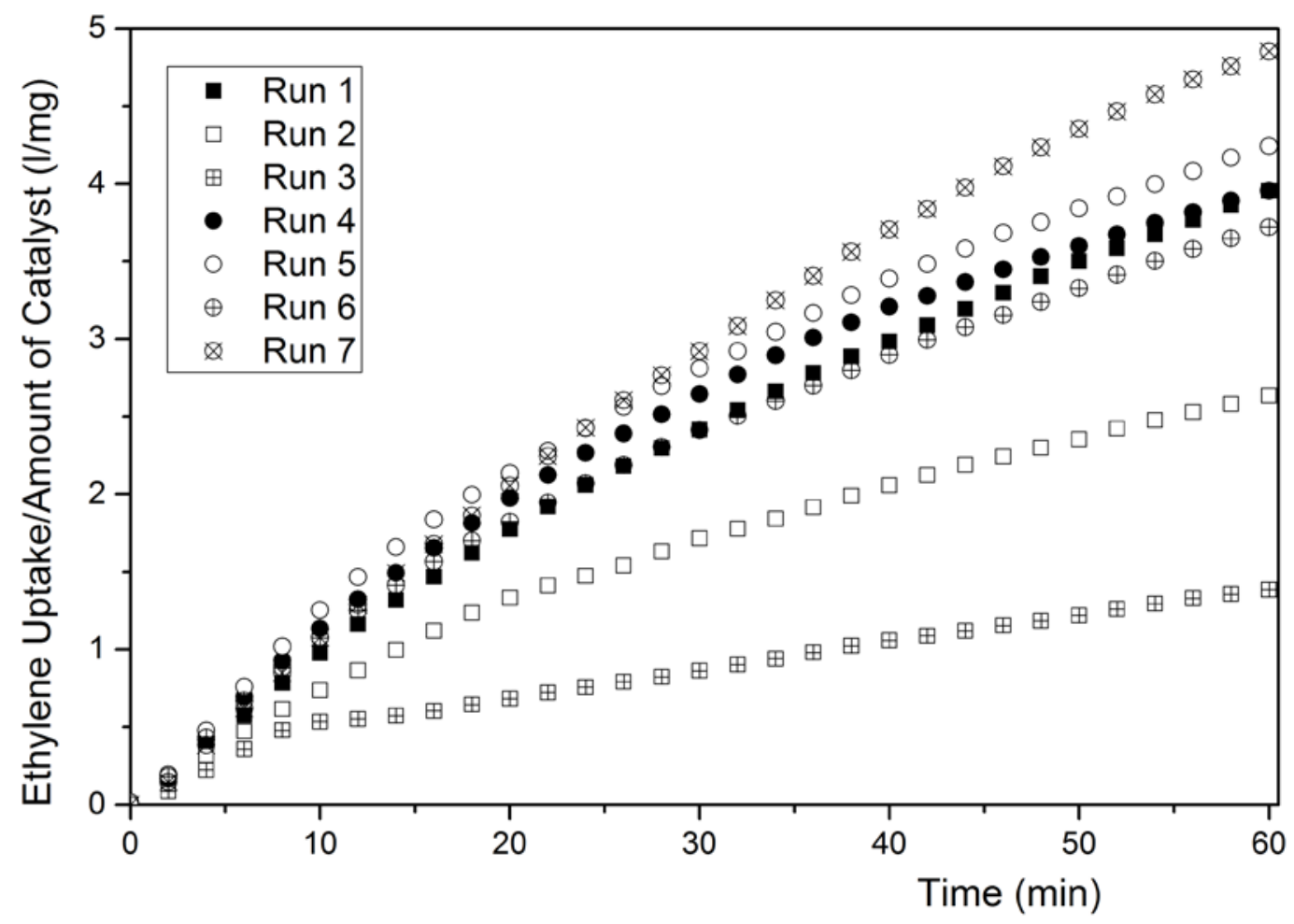

Figure 2: Ethylene uptake normalized by the amount of catalyst used as a function of polymerization time for the polymers listed in table 1.

From the ethylene uptake profile of figure 2 it is clear that the BHT has a significant effect on the catalytic system. When the catalyst is activated with MAO only, the TMA present in equilibrium with the polymeric form can interact with the catalyst leading to non-active species towards the ethylene polymerization. This effect becomes more pronounced when the contact time is longer than few minutes. If the amount of TMA present in the MAO is reduced using the BHT, the formation of the non-active species is considerably suppressed in the time range studied; this is evident by the ethylene uptake profile that becomes similar to the activation with MAO without delay in the catalyst activation. However, the MAO used as scavenger, having remaining TMA, can still interact with the catalyst leading to non-active species toward the polymerization of ethylene. If the BHT is used in combination with all the MAO used in the polymerization, the ethylene uptake profile is higher compared to all the other profiles. Having longer activation time is sometime a fundamental industrial requirement due to long residence time of the activated catalyst in the production plants. The use of BHT during the activation step can overcome the drawback of this catalyst when activated with MAO. In addition, the BHT is an antioxidant used sometime into polymers to avoid degradation during the processing step(s). Considering the intimate dispersion of BHT into the polymer particles (polymerization with the presence of the antioxidant), the amount needed to avoid 
polymer degradation can be even lower if compared with the normal and more expensive procedure to add the antioxidant after the polymerization.

Rheological characterization of the polymers produced shows low starting value (at $t=0$ ) of the elastic modulus (well below $1 \mathrm{MPa}$ ) and slow modulus build-up with time for all the polymers summarized in table 1 (Figure 3). According to previous reported findings, the rheological response is related with a reduced number of entanglements in the amorphous phase of the nascent powder. ${ }^{[9]}$

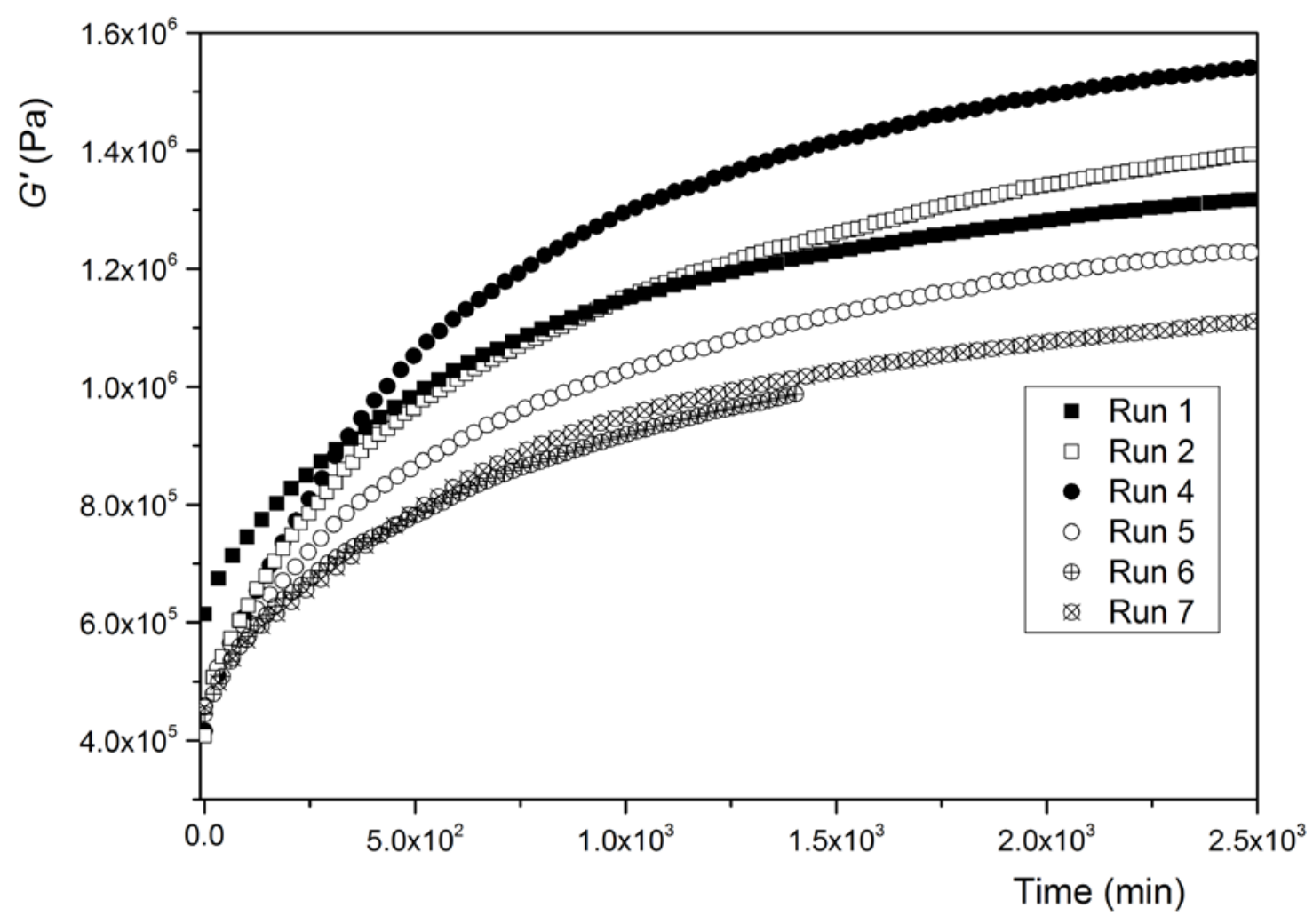

Figure 3: Elastic modulus as a function of time for the polymers listed in table 1.

To recall, rheology can also be used to estimate molecular weight and molecular weight distribution of polymers by measuring the viscoelastic functions (Elastic G' and Loss G" Moduli) as a function of angular frequency and fit the data with a suitable mathematical method. ${ }^{[16]}$ The mathematical expression to convert the relaxation modulus $(G(t))$ to the molecular weight and molecular weight distribution is given in equation 1 :

$$
G(t)=G_{N}^{0}\left(\int_{\ln M_{e}}^{\infty} F^{\frac{1}{2}}(t, M) w(M) d(\ln M)\right)^{2}
$$

Where $G_{N}^{0}$ is the rubbery elastic plateau value $\left(\sim 2.0^{*} 10^{6} \mathrm{~Pa}\right), M_{e}$ is the mass between consecutive entanglements (1900 g/mol), $w(M)$ represents the probability of finding chains 
with molar mass between $M$ and $M+d M, F(t, M)$ corresponds to the reptation memory function $F=\exp \left(-t / t_{d}\right)$, where the phenomenological relation for the reptation time $t_{d} \sim M^{3.4}$ has been used.

A summary of the frequency sweep tests of the polymers synthesized is showed below.

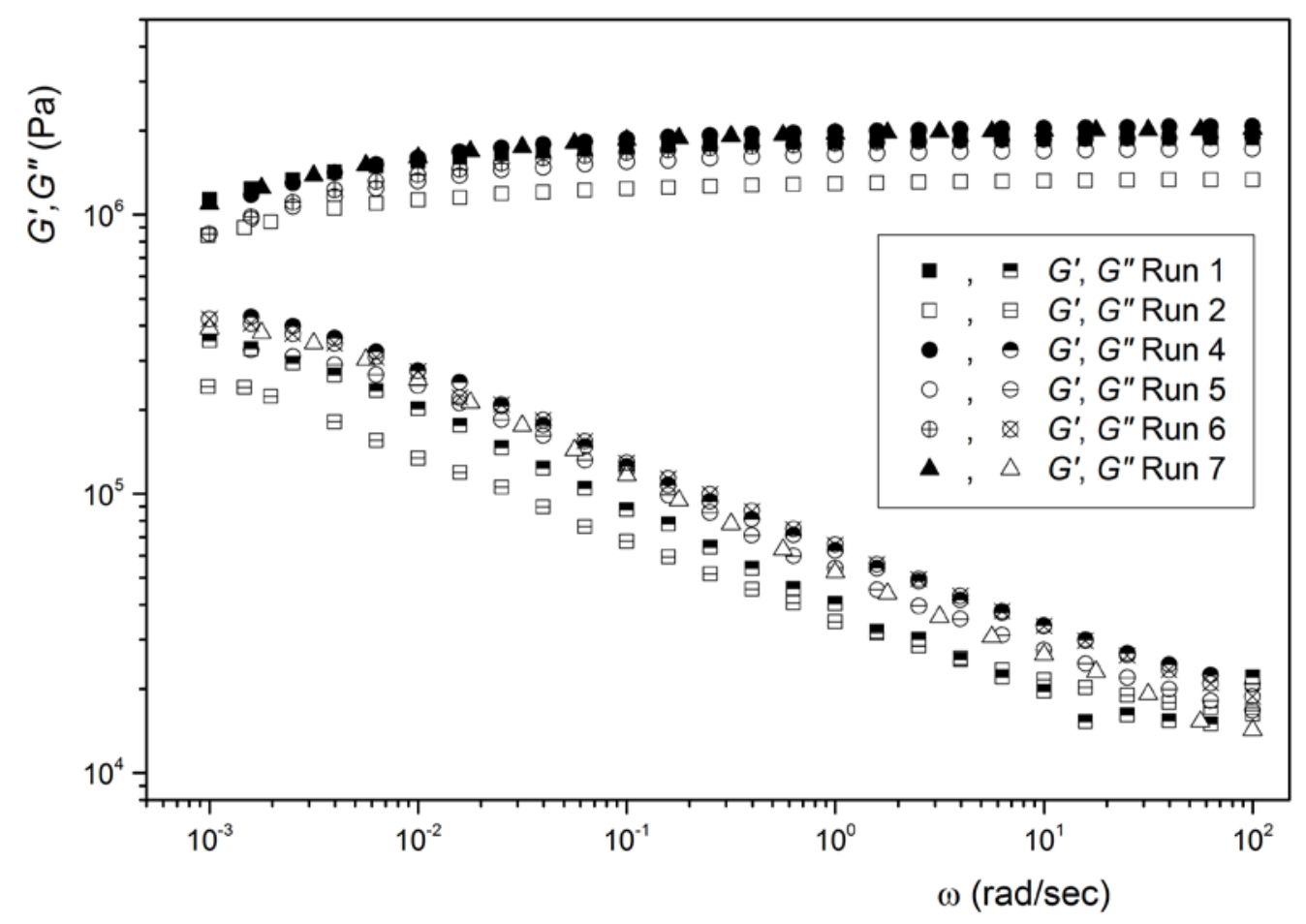

Figure 4: Elastic and loss moduli as a function of angular frequency $(\omega)$ for the polymers of table 1.

The estimation of weight-average molecular weight $\left(M_{\mathrm{w}}\right)$ and molecular weight distribution (MWD), are in agreement with previous reported findings and prove the synthesis of polymers with a molecular weight exceeding $6.6^{*} 10^{6} \mathrm{~g} / \mathrm{mol}$ for all the polymers listed in table 1. In the reaction conditions explored, the values of MWD are in agreement with polymerizations performed using a single-site catalyst. Differences between the estimations of the molecular weights of the polymers produced can still be discerned. Runs 1-2 have larger $M_{\mathrm{w}}$ (synthesis without BHT) compared with Runs 4-7 (synthesis with BHT). According to previous study, this is related with a second active species capable to produce longer chains generated upon activation with MAO (due to an interaction between the TMA present in equilibrium with the polymeric form and the catalyst). The use of BHT during the activation step scavenges the TMA in the MAO used to activate the catalyst, avoiding the formation of secondary active species. ${ }^{[20]}$ The broadening of the MWD of Run 2 compared with Run 1 is in agreement with the 
hypothesis of the formation of additional species during the long activation time (15 minutes). In contrast with the activation without BHT, no significant differences can be found comparing the $M_{\mathrm{w}}$ and MWD of Runs 4-7. This further supports the detrimental effect of the TMA during the activation step; the TMA-free MAO can allow the formation of (potentially) only one active species even increasing the delay during the activation step.

In table 2 are summarized the ethylene polymerization data using the FI catalyst activated with PMAO and MMAO12 increasing the activation time.

Table 2 Ethylene polymerization data using PMAO and MMAO12 aluminoxanes in different activation procedure.

\begin{tabular}{|l|l|l|l|}
\hline Run & Co-catalyst & $\begin{array}{l}\text { Activation } \\
\text { Time } \\
(\mathrm{min})\end{array}$ & $\mathrm{Rp}^{a}$ \\
\hline 8 & PMAO & 0 & 900 \\
\hline 9 & PMAO & 10 & 2850 \\
\hline 10 & PMAO & 30 & 2200 \\
\hline 11 & MMAO12 & 0 & 1150 \\
\hline 12 & MMAO12 & 10 & 2500 \\
\hline 13 & MMAO12 & 30 & 3000 \\
\hline
\end{tabular}

Other conditions: Al/Ti molar ratio, 1850; catalyst, $6.2 \pm 0.2 \mu \mathrm{mol}$; temperature, $10{ }^{\circ} \mathrm{C}$; reaction volume, $750 \mathrm{ml}$ of toluene; reaction time, $60 \mathrm{~min}$; ethylene pressure, $1.1 \mathrm{~atm}$. $\mathrm{kg}_{\mathrm{PE}} / \mathrm{mol}_{\mathrm{Ti}} *$ atm*hour.

For the catalytic system FI/PMAO (Runs 8-10), it is not detrimental for the catalyst activity to delay the injection of the activated catalyst in the reactor vessel containing the monomer. Figure 5 summarizes the data in table 2 for the PMAO (Runs 8-10). 


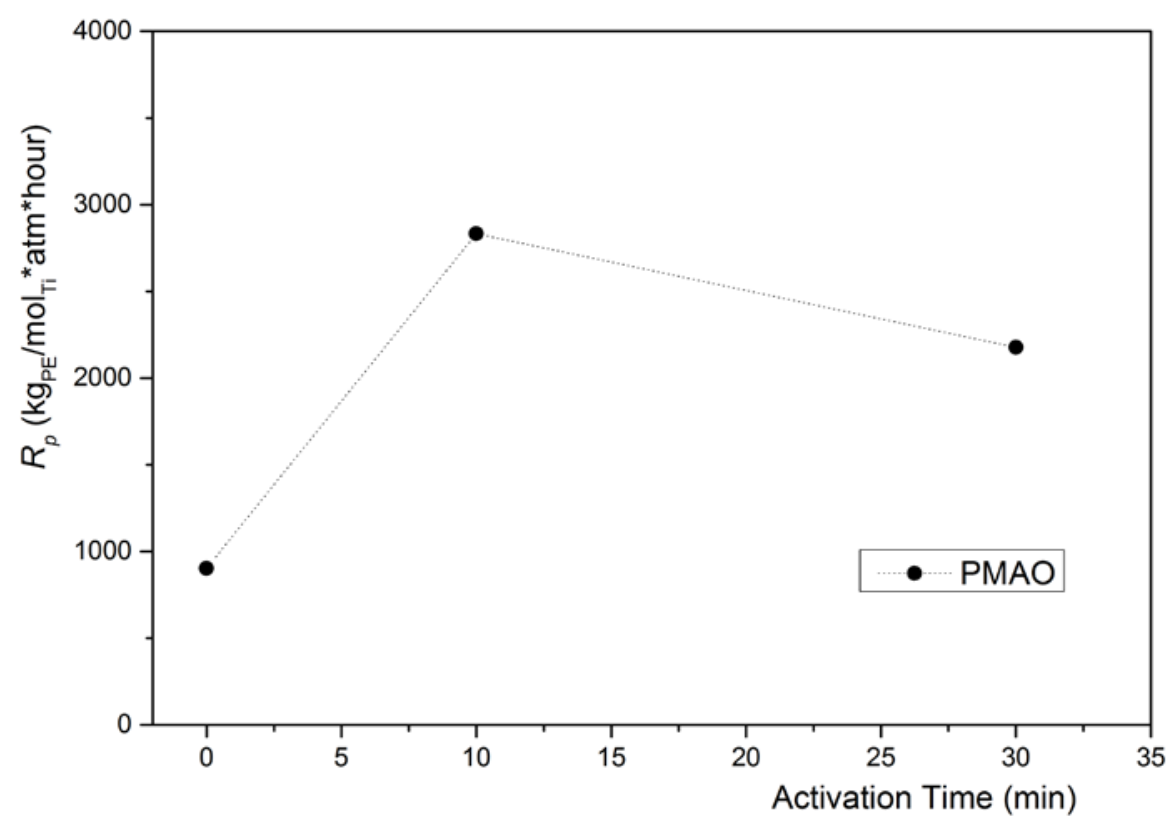

Figure 5: Catalyst activity as a function of activation time for the activation using PMAO. The dotted line is drawn for eye guidance only.

For the catalytic system FI/MMAO12 the catalyst activity increases by increasing the activation time up to 30 minutes. Figure 6 summarizes the data in table 2 for the MMAO12 (Runs 11-13).

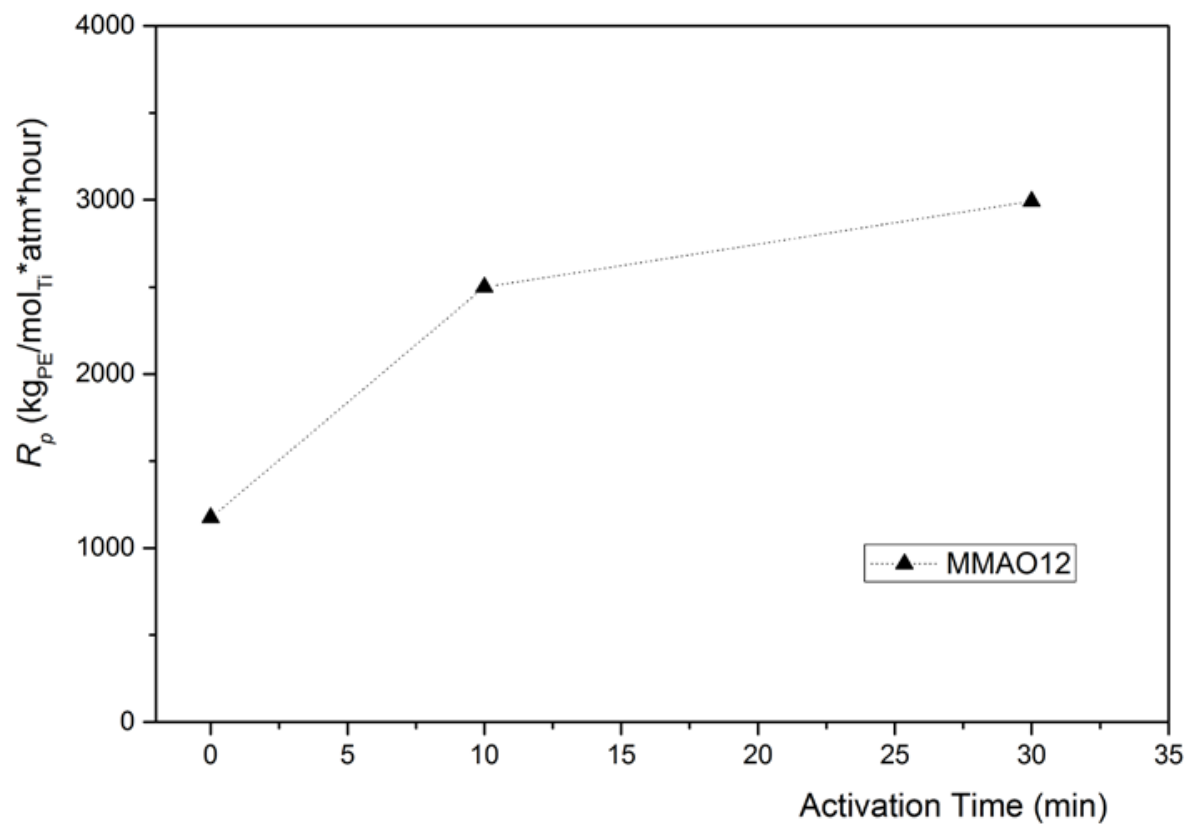

Figure 6: Catalyst activity as a function of activation time for the activation using MMAO12. The dotted line is drawn for eye guidance only.

Contrary to the activation with MAO, for the catalytic systems FI/PMAO and FI/MMAO12 it is beneficial to leave the catalyst in contact with the aluminoxane for a 
certain time. The activation process is in general dependent on the structure of the catalyst and co-catalyst. In comparison to the activation by MAO, possibly the PMAO and MMAO12 have slower capabilities to generate the active species during the activation step. However, other catalyst deactivation processes can occur at longer activation times (over 30 minutes).

Several potential reasons could be invoked to explain the difference in the activation capabilities. Differences in the cage structure, different alkyl groups involved in the alkylation and/or formation of the cationic active site, different kind of TMA present (bounded and free TMA) and their relative concentrations, difference in the polarity of the media etc. can be argued. However, the discrimination and the study of these effects can be particularly difficult and it is outside the scope of this study.

\section{Conclusions}

The activation of the bis[ $N$-(3-tert-butylsalicylidene)pentafluoroanilinato] titanium (IV) dichloride catalyst has been investigated using the MAO in different activation procedures. The BHT has also been used in combination with the MAO to test the effect of the absence of TMA during the activation step. Ethylene uptake profile shows a reduction of monomer consumption when the TMA is present in the activation step and during the polymerization. The reduction of ethylene uptake is even more pronounced when the catalyst activation time increases up to 30 minutes in the presence of TMA. These reductions in the ethylene consumptions are probably due to catalyst deactivation processes leading to catalyst modification into non-active species. By removing the TMA from the activation step, using the BHT, the catalyst does not undergo deactivation processes even by increasing the activation time in the time range explored.

In agreement with the rheological characterization of the polymers produced, the catalyst is capable to promote the synthesis of disentangled UHMWPE in all the conditions used in table 1 . The synthesized polymers can be solid-state processed into high modulus/strength tapes and fibers having high tenacity ( $>4.3 \mathrm{~N} / \mathrm{tex})$ and high modulus ( $>200 \mathrm{~N} / \mathrm{tex}) .{ }^{[12]}$ The values of $M_{\mathrm{w}}$ of the polymers produced when the TMA is present in the activation step are higher due to the presence of a secondary species. This secondary species is also responsible for the broadening of the MWD of the polymers synthesized when the catalyst is activated with MAO, compared to the polymers synthesized using TMA-free MAO. The use of BHT in the activation step avoids the formation of the secondary species with a 
consequent reduction of the values of $M_{\mathrm{w}}$ and MWD.

The catalyst can be successfully activated in the polymerization of ethylene also with PMAO and MMAO12. A lower catalyst activity is recorded when compared with the activation with MAO. Contrary to the activation with MAO (in the absence of BHT), for PMAO and MMAO12 it is beneficial to increase the activation time with the catalyst. The lower catalyst activity for PMAO and MMAO12 in combination with the possibility to increase the activation time suggest that these two aluminoxanes are less capable (or with a slower rate) to generate the active site when compared with MAO. However, differences in the content and the kind of the TMA present must be taken into consideration.

[1] J. Saito, M. Mitani, S. Matsui, M. Sugi, Y. Tohi, T. Tsutsi, T. Fujita, M. Nitabaru, H. Makio (Mitsui Chemicals, Inc.). Eur. Patent 0,874,005 A1, 1998, October 28.

[2] S. Matsui, T. Fujita, Catal. Today, 2001, 66, 63-73.

[3] H. Terao, S. Ishii, J. Saito, S. Matsuura, M. Mitani, N. Nagai, H. Tanaka, T. Fujita, 2006, Macromolecules, 39, 8584-8593.

[4] L. Caporaso, M. Loria, M. Mazzeo, K. Michiue, T. Nakano, T. Fujita, L. Cavallo, 2012, Macromolecules, 45, 8588-8597.

[5] H. Makio, N. Kashiwa, T. Fujita, Adv. Synth. Catal. 2002, 344, 477-493.

[6] H. Makio, H. Terao, A. Iwashita, T. Fujita, Chem. Rev. 2011, 111, 2363-2449.

[7] M. Mitani, J. Mohri, Y. Yoshida, J. Saito, S. Ishii, K. Tsuru, S. Matsui, R. Furuyama, T. Nakano, H. Tanaka, S. Kojoh, T. Matsugi, N. Kashiwa, T. Fujita, J. Am. Chem. Soc. 2002, 124, 3327-3336.

[8] S. Rastogi, Y. Yao, S. Ronca, J. Bos, J. van der Eem, Macromolecules, 2011, 44, 5558-5568.

[9] S. Rastogi, D.R. Lippits, G.W.M. Peters, R. Graf, Y. Yao, H.W. Spiess, Nat. Mater. 2005, 4, 635-641.

[10] P. Smith, P.J. Lemstra, J. Mat. Sci. 1980, 15, 505-514.

[11] P. Smith, P.J. Lemstra, Makromol. Chem. 1979, 180, 2983-2986.

[12] D. Romano, N. Tops, E. Andablo-Reyes, S. Ronca, S. Rastogi, Macromolecules, 2014, 47, 4750-4760.

[13] V. Busico, R. Cipullo, F. Cutillo, N. Friederichs, S. Ronca, B. Wang, J. Am. Chem. Soc. 2003, 125, 12402-12403.

[14] S. Ronca, D. Romano, G. Forte, E. Andablo-Reyes, S. Rastogi, Adv. Polym. Tech. 2012, 31, $193-204$.

[15] C.W. Macosko, Principle, Measurements and Applications; Rheology, Wiley-VCH: New York, 1994; Chapter3, pp 121-125.

[16] D. W. Mead, J. Rheo. 1994, 38, 1797-1827.

[17] H. Makio, T. Fujita, Accounts Chem. Res. 2009, 42, 1532-1544.

[18] K.P. Bryliakov, E.A. Kravtsov, D.A. Pennington, S.J. Lancaster, M. Bochmann, H.H. Brintzinger, E.P. Talsi, Organometallics, 2005, 24, 5660-5664.

[19] H. Makio, T. Fujita, Macromol. Symp. 2004, 213, 221-233.

[20] D. Romano, E.A. Andablo-Reyes, S. Ronca, S. Rastogi, J. Polym. Sci. A1, 2013, 51, 1630-1635. 